\section{Technical boundless optimism}

David E. H. Jones

Nano! Remaking the World Atom by Atom. By Ed Regis. Little, Brown/Bantam. Pp. 307. \$23.95, £16.99.
THE boundless-optimism school of technical forecasting covers a wide spectrum. The more realistic end features such marvels as nuclear electricity too cheap to meter (remember that?) and computers that understand spoken English. At the manic end we find space colonies located at Lagrangian points, the terraforming of neighbouring planets, and robots in convincing human form. A promising newcomer has recently appeared somewhere in the middle of this spectrum. It is called nanotechnology.

Nanotechnology must be carefully distinguished from microtechnology. The latter, which could operate down to the micrometre scale, is simply the manufacture and use of extremely small mechanisms - microscopic lathes, motors and so on. Nobody has got very far with it, and it might or might not be much use, but the theory seems unexceptionable. As early as 1959 Richard Feynman pointed out that a lathe could be used to make the parts for a smaller lathe; this could make the parts for one smaller still, and the very obvious limit. Modern photofabrication can get quite a way in one step, by exploiting the techniques developed for integrated-circuit manufacture. Micrometre-sized microphones and forcetransducers have already been etched out in this way.

Nanotechnology takes this idea to its ultimate extreme - the direct assembly of components and structures atom by atom. Its prophets assert that anything whatever could - and once the trick has been perfected, will - be made by atomic assembly. The nanotechnological John the Baptist is Eric Drexler of the Massachusetts Institute of Technology. He has been crying in the wilderness since at least 1980 and has acquired a group of enthusiastic disciples. Ed Regis is one of them, and Nano! is his account of the revelation so far.

Nano! is a dramatic work. It is a breathless, exhaustingly complete, desperately confusing, utterly uncritical, thought-bythought, meeting-by-meeting, paper-bypaper account of Drexler, his life, times and growing missionary conviction that nanotechnology will lead us to the promised land. As a story, a personal biography of an imaginative and committed individual, it has its own interest and process could continue downwards to no human appeal. As an account of a clique of enthusiasts and their vision of the future, it sheds an intriguing light on the psychology of technical boundless optimism.

The vision, roughly, is this. In the nanotechnological world, everything will be made by tiny programmable 'assemblers', about the size of bacteria, whose exact specification cannot yet be given. Swimming around in a sort of water-bath, universal panacea. How, for example, would it reverse the spreading deserts or halt the population explosion? But the big question, of course, is whether it would work. Regis provides five main arguments in its favour: Feynman has endorsed the feasibility of arbitrarily small machines; scanning tunnelling microscopes have already manipulated single atoms successfully; the normal syntheses of chemistry in effect manipulate single atoms, and do it in trillionfold parallel into the bargain; bacteria function and replicate, so it must be possible; and the uncertainty principle and brownian motion do not seem to worry them.

These arguments deserve closer scrutiny than Regis gives them. How small can a machine, or more precisely a machine tool, be made? It has two essential com-

के ponents: a 'cutter' to act on the workpiece, and a 'frame' to act as a reference, move the cutter around the workpiece and absorb the cutting forces. In a standard engineering machine tool, such as a numerically controlled lathe or milling machine, the cutter is just that; but the analysis applies more generally.

A fundamental requirement of any machine tool is that the frame must be much bigger than the cutter. In a lathe or milling machine, the cutter has to apply its force over such a small area that the local stress deforms or shears the workpiece. The reaction to this force is of the same magnitude, but it must be absorbed by the frame with a deflection that is small compared with the desired resolution of the cutting action. The frame therefore needs a much larger cross-section than the cutter, so that this force represents a very small stress within it. The tool can then impose any pattern of deformation on the workpiece without change or damage to itself. Armed with overwhelming force, it can operate in a completely dictatorial engineering mode.

This requirement for a frame much bigger than the cutter extends to nonmechanical tools as well. A photofabricator, for example, has an optical 'cutter'. To focus as sharply as a wavelength, its lens (by the familiar laws of optics) must be many wavelengths across. To work as an atomic manipulator, a scanning tunnelling microscope might use an electrical potential as its 'cutter'. It would hold and release atoms by applying and removing a strong electric field across them. For this to work, its stylus and stage must be much larger than an atom. A stylus (say) a million atoms long will sustain at most a field one millionth of the one it imposes on the target atom. Now imagine making the microscope smaller and smaller, shrinking it down to a Drexler assembler and even 
beyond. With every tenfold reduction in size, its internal field rises tenfold. It could perhaps reach the micrometre scale in working order. But by the time it approached the size of the target atom, the field within it would be disrupting its own atoms, and its operation would fail. Once a machine tool is so tiny that its frame cannot be much larger than its cutter, it can no longer function as a tool. Engineering must give way to chemistry, the science of single atoms or groups of atoms acting on each other.

\section{Chemical constraints}

And chemistry is a much humbler science than engineering. No chemist can impose an arbitrary design on his raw material, as an engineer can on his. Chemistry is a matter not of dictatorial programming but of cunning and luck and selection. It has to seek out favourable conditions and suitable reagents. It tries to set up molecular encounters that, by their own nature, their random thermodynamic shuffling towards the most accessible local energy minimum and global entropy maximum, will form the desired product. A great many substances and material forms can be made this way, but an infinitely greater number cannot. The successes of chemistry cannot be represented as an argument for nanotechnology.

Indeed, the nanotechnologists do not seem to realize the chemical obstacles in their path. To break a chunk of raw material into its component atoms needs a lot of energy - at least the latent heat of vaporization. And the single atoms when you have them cannot just be picked out and pushed around like so many marbles. When D. M. Eigler and E. K. Schweizer wrote the IBM logo in single atoms in a scanning tunnelling microscope, they used inert xenon atoms in an ultra-high vacuum at liquid helium temperature, dodging the main physical and chemical problems; and even then the atoms stayed put on their substrate only in certain positions and spacings. Single atoms of more structurally useful elements, at or near room temperature, are amazingly mobile and reactive. They will combine instantly with ambient air, water, each other, the fluid supporting the assemblers, or the assemblers themselves. And even if you manage to assemble them into some masterpiece of nanostyling, you had better be sure that it is everywhere an energy-minimum on the atomic scale. Otherwise it won't be stable. Its atoms will recrystallize and diffuse and react towards their own guess at such a minimum. You will have terrible problems with chemistry.

Ah yes, says the nanoenthusiast, but what about biochemistry? What about bacteria, those living exemplars of selfreplication and construction on the micro and nano scales? If chemistry is so restricted and troublesome, how do they manage? The answer, I think, is by extraordinary chemical specialization. Life seems to be an exercise in doing the best you can with the minimum of information. The genetic code does not specify the whole structure of an organism atom by atom. It provides a recipe that, if followed without too many blunders, produces a functioning creature capable of handing on much the same recipe. Over evolutionary time, those creatures have prospered whose recipes are the simplest and most robust for what they do.

The same constraint appears in the software of living behaviour. Termites, for example, collectively construct huge nests, in the same sort of way that Drexler imagines his assemblers building a telephone or a dishwasher. Philip Morrison analysed this process instructively in 1979 . He showed that the nest results from a mass of termites all blindly following an algorithm with a few simple rules. Nowhere is there any plan or blueprint and no two nests are alike, but the structures always work as nests. It is clear that termite nests, like the termites themselves, have evolved as minimum-information structures. Termites whose nests required a more complicated algorithm failed to survive. Could termites be modified by gen-etic engineering to assemble, not crude variable nests but (to use Morrison's example) identical working astronomical telescopes? No: the enormous algorithm required would simply overwhelm them.

So it is wrong-headed to argue that, because a creature as small as a bacterium can reproduce itself, therefore a nanotechnological assembler can also replicate itself and, furthermore, will have enough spare capacity to replicate anything else you care to imagine. The chemistry of life centres on some highly specialized organic chemicals: DNA to carry information, a set of amino acids from which to form the proteins that double both as catalysts and as structural material, sugars and fats as energy-stores, and so on.

It is reasonable to guess that this particular biochemistry has survived and stabilized because it operates effectively with the absolute minimum of information. It permits and constitutes the ultimate in elegant programming. To claim that nanotechnological assemblers could work the same chemical trick with chemically unsubtle materials such as aluminium and diamond, and replicate themselves with enough spare capacity to replicate anything else as well, is equivalent to asserting that life itself could be made to work in these materials, and with the added handicap of a vast overhead of arbitrary information.

This, of course, is chemically incredible. Nanotechnology will not be able to avail itself of the sparse, elegant, specialized programming evolved by biochemistry. It will have to work by engineering. Its assemblers will somehow have to pack not only scanning tunnelling microscope technology or its equivalent into their bacterial dimensions, but the terabytes of brute-force coding and processing power needed to specify themselves and their products atom by atom. And I don't believe it will fit.

Regis's final argument concerns the two imprecisions that lurk in the nanodomain, the uncertainty principle and brownian motion. The uncertainty principle, set up and knocked down several times in Nano!, is rightly discounted. It doesn't stop atoms having a location or molecules having a structure; it merely makes their edges a bit fuzzy. Only at the subatomic level would it start to give serious trouble.

Brownian motion, however, cannot be dismissed so lightly. It raises a fundamental question that the nanoethusiasts seem to have ignored: that of entropy. Formally, this damns their entire project. Above absolute zero, entropic considerations prevent even a single crystal, energyminimum that it is, from ever being perfect. By the same token, the purest product of nanotechnology could never exist at room temperature "with no atom out of place", as Drexler dreams. But let us not be so unforgiving. Crystals are impressively regular structures despite their dislocations, and cells have learnt to live with imperfections in their DNA. A sound energy-minimized nanotechnological design should be able to tolerate a certain proportion of misplaced atoms without disaster. What constraints does thermodynamics place on its assembly?

\section{Demon assemblers}

The very first nanotechnologist was Clerk Maxwell's celebrated Demon, proposed in 1871. This was the little fellow who sat in the connecting pipe between two reservoirs of gas, watching the molecules pass between them. By blocking all molecules travelling one way, but allowing free passage to those going the other way, the Demon could build up a pressure difference across the two reservoirs. By letting fast molecules go one way and slow ones the other, he could build up a temperature difference. Either of these could be used to generate mechanical power, so the Demon could extract energy from a thermal system at one temperature - in contravention of the second law of thermodynamics.

Maxwell's Demon was a puzzle to physicists for many years, but has been well exorcized by now. The first blow was struck by Leo Szilard in 1929, when he pointed out that the Demon needed to obtain information about the molecules. $\mathrm{He}$ argued that the entropy generated by this interrogation exactly cancelled the reduction of entropy that could be achieved by exploiting the answer. This 
elegant analysis initiated our modern identification of information with negative entropy; it has since been taken further and made part of computing theory. It is now clear that Maxwell's Demon cannot possibly work.

Nanotechnological assemblers look suspiciously like Maxwell's Demons. Drexler's argument that they are "programmed, like infecting a bacterium with a virus", evades important questions about their thermodynamics and information flow. How do the assemblers get their information about which atom is where, in order to recognize and seize it? How do they know where they themselves are, so as to navigate from the supply dump to the correct position in which to place it? How will they get their power, for comminution to single atoms, navigation and, above all, for massive internal computing? How will they dispose of the entropy of their operations, and how much will they have to dispose of? The best modern computers still dissipate about $10^{12}$ times as much entropy as is theoretically needed by their information flow, so even allowing for improvements it could be quite a lot. Until these questions are properly formulated and answered, nanotechnology need not be taken seriously. It will remain just another exhibit in the freak-show that is the boundlessoptimism school of technical forecasting. $\square$

David E. H. Jones is in the Department of Chemistry, University of Newcastle upon Tyne, Newcastle upon Tyne NE1 7RU, UK.

\section{Proving the rule}

\section{Peter Lipton}

Killing Time: The Autobiography of Paul Feyerabend. By Paul Feyerabend. University of Chicago Press: 1995. Pp. 181. £18.25, \$22.95.

PAUL Feyerabend, who died in February last year, was the gadfly of the philosophy of science. Born and raised in Vienna, he fought for Germany during the Second World War, suffering bullet wounds to the spine that left him without effective use of his legs for the rest of his life. After the war, he returned to Vienna to study physics, with extensive philosophy and operatic singing on the side. Although much of Feyerabend's doctoral work was on a technical problem in classical electrodynamics, he ended up writing a philosophy thesis, for which he received a doctorate in 1951. He was then awarded a British Council scholarship to study with Wittgenstein in Cambridge, but had to find a different supervisor when Wittgenstein died the same year. He chose Karl Popper and became for a short time a card-carrying falsificationist. Soon, how- ever, he rejected Popper's philosophy of science with a vengeance, taking it as a model of how philosophy ought not to be done. In the long run, few contemporary philosophers of science managed to avoid Feyerabend's scorn.

Feyerabend's writings, best represented by Against Method (New Left Books, 1975), is a verbal blitzkrieg: aggressive, fast-moving and powerful. It is also enter-

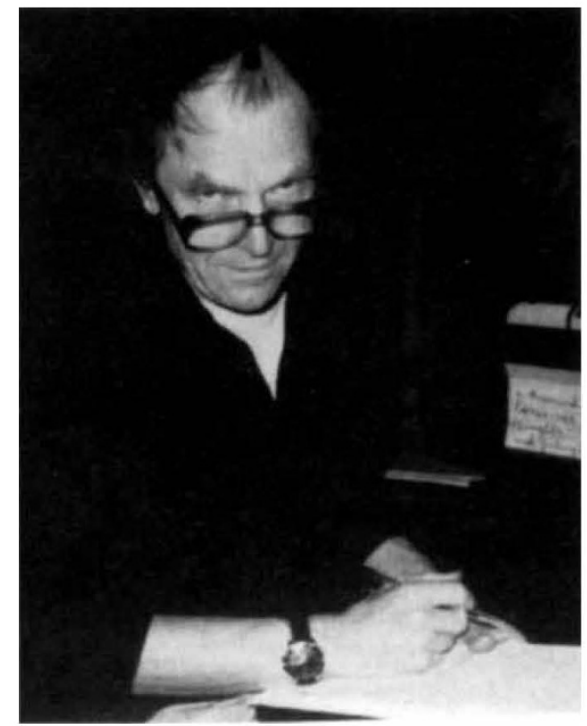

Feyerabend: science's worst enemy?

taining, infuriating and sometimes offensive. His primary target is the traditional philosophical project of explaining and defending scientific method. His strategy is to argue that any rules of scientific practice must be either so weak as to exclude nothing or persistently violated by the best scientific work. As he characteristically puts it: "The only principle that does not inhibit progress is: anything goes".

Feyerabend's attack on the very possibility of a non-trivial account of scientific practice comes in two stages. First he claims that the most basic methodological rules proposed by various philosophers of science were in fact broken in historical episodes that those philosophers themselves regard as models of science at its best, episodes that brought about the Copernican revolution, kinetic theory and quantum mechanics. The rules allegedly broken include those requiring that a new theory should be consistent with its predecessors or at least with the available evidence, that it should avoid ad hoc hypotheses and even that it should not be self-contradictory. The poor philosophers are themselves caught in a contradiction between the rules they invent and the science they admire.

The second stage of Feyerabend's attack on method deploys a variety of arguments to show that the violation of standard rules is not only a historical fact but is also essential to scientific progress. The arguments depend on two radical claims: that data are never independent of theory and that competing theories may be logically incommensurable. Feyerabend uses these claims to argue that a scientific theory cannot be evaluated by testing it against experiment and observation alone, but only by confronting it with radical alternatives. This case for theoretical pluralism gives Feyerabend the excuse to take his attack on the philosophers into overdrive, applying it to science itself. In this scheme, effective criticism involves confronting scientific theories with all sorts of nonscientific world views, however bizarre. And there is no presumption that conventional science ought to win out in the end.

Feyerabend's defence of voodoo is unlikely to impress many readers of Nature, but his case against the philosophical project of defining rules of scientific practice has considerable force. $\mathrm{He}$ certainly scores palpable hits against particular methodological prescriptions, especially those of a Popperian stripe. This does not, however, prove the negative. Showing the failures of one or another account of scientific method is not the same as showing that no successful account is possible. Moreover, even if scientific research does not lend itself to a rule-based description, the possibility of a general account remains because rules are not the only source of generality.

On this last point, Thomas Kuhn's Structure of Scientific Revolutions (University of Chicago Press, 1962) provides an excellent foil to Against Method. Kuhn and Feyerabend hold many radical views in common: scientific theories may be incommensurable, there are no facts independent of theory, and scientific research is not governed by rules. But the perceived absence of rules does not lead Kuhn to abandon the idea of a general account of scientific research. Instead, he replaces rule-based accounts of science with one based on the power of concrete problem-solutions or exemplars to guide research in the absence of explicit rules. Feyerabend's epistemological anarchy is not the only alternative to the view that there could be a rule-book of good scientific practice.

Feyerabend's autobiography includes some philosophy, but it is mostly an indiscreet reminiscence of the life and times of a wild philosopher. It makes entertaining reading and provides plenty of material about Feyerabend's early life for anyone who wants to attempt to give a psychological explanation for his later intellectual excesses. Readers interested in the provocative arguments themselves, however, are better off thickening their skins and reading Against Method.

Peter Lipton is in the Department of History and Philosophy of Science, University of Cambridge, Free School Lane, Cambridge CB2 3RH, UK. 